\title{
Intraarterial chemotherapy as the first-line therapy in penile cancer
}

\author{
P-H Chiang *,1,3, C-H Chen ${ }^{1,2,3}$ and Y-C Shen ${ }^{1}$ \\ ${ }^{1}$ Department of Urology, Kaohsiung Chang Gung Memorial Hospital and Chang Gung University College of Medicine, 123, \\ Ta-Pei Road, Niaosung, Kaohsiung 83301, Taiwan and ${ }^{2}$ Graduate Institute of Medicine, College of Medicine, Kaohsiung Medical \\ University, Kaohsiung, Taiwan
}

Background: Limited literature on the role of intraarterial chemotherapy as first-line therapy for penile squamous cell carcinoma is available.

Methods: From 2005 to 2013, a total of 12 patients with various stages of penile squamous cell carcinoma received intraarterial chemotherapy. The chemotherapeutic agents used were methotrexate, mitomycin C, bleomycin, cisplatin, and 5-fluorouracil. Surgery was followed by the tumour responses.

Results: An objective tumour response was noted in 10 of 12 patients (83\%, 4 complete responders and 6 partial responders). In node-negative patients $(n=7)$, the response rate was 100\% (4 complete responders and 3 partial responders). Even in advanced penile squamous cell carcinoma with nodal invasion, a response rate of $60 \%$ could be achieved. Grade 2 anorexia was the most frequent chemotherapy-related toxicity and no toxic death was noted. Recurrence-free survival was significantly better in patients without lymph node invasion (log-rank test, $P=0.041$ ).

Conclusions: Neoadjuvant intraarterial chemotherapy displayed excellent responses for penile squamous cell carcinoma. This therapy could effectively shrink the tumour burden or even achieve complete response before surgery. It could be used as first-line strategy for penile cancer treatment because of low toxicity.

Squamous cell carcinoma (SCC) of the penis is a rare but ominous disease in men. The incidence of penile SCC is relatively low in Taiwan and the Western countries. However, $\sim 30 \%$ of patients with penile SCC present with advanced stage at initial diagnosis (Culkin and Beer, 2003). For a large localised tumour, total or partial amputation of the penis with a healthy margin of 5-10 $\mathrm{mm}$ has been considered to be the preferred option of treatment according to the guideline (Pizzocaro et al, 2010). However, there may be remarkable impact on psychosexual aspect and quality of life after surgery because of shortening of penile length, especially in sexually active patients. Thus, some experts try to seek the penissparing modality with concomitant oncological control.

Intraarterial (IA) chemotherapy is a known alternative choice for cancer treatment. The rationale of IA chemotherapy is to deliver a high concentration of anticancer agents to target organ with less systemic drug exposure. Clinically, this method has been applied in the treatment of lip cancer, oral cancer, liver cancer, breast cancer and so on with encouraging results (Wu et al, 2007, 2008; Tanaka et al, 2013; Zhang et al, 2013). However, in the treatment of penile SCC, the literature on IA chemotherapy remains limited and fragmented. In this study, we have reported 12 patients with various stages of penile SCC treated by IA chemotherapy. To our knowledge, this is the largest report of data on IA chemotherapy in the treatment of penile SCC.

\section{MATERIALS AND METHODS}

Patient selection. We retrospectively reviewed all the patients with penile SCC from 2005 to 2013. Only patients receiving IA chemotherapy were included. For tumour staging, detailed physical

*Correspondence: Professor P-H Chiang; E-mail: tuoa480713@yahoo.com.tw

${ }^{3}$ These authors contributed equally to this work and serve as co-first authors.

Received 14 April 2014; revised 3 June 2014; accepted 18 June 2014; published online 24 July 2014

(c) 2014 Cancer Research UK. All rights reserved 0007-0920/14 
examination and imaging studies (chest radiography and CT scan) were performed to assess the tumour status. This study was reviewed and approved by the Human Investigation Review Committee of our hospital. Informed consent was obtained from all patients.

Intraarterial chemotherapy regimens and protocol. After admission, routine haematologic and biochemical tests were performed. Before injection of chemotherapy regimens, the IA catheter was placed first by radiologist. Briefly, a 4 - or 5-F pigtail catheter was inserted into the lower abdominal aorta with the tip at the level above aortic bifurcation and below the inferior mesenteric artery. The infused regimens consisted of methotrexate $\left(110 \mathrm{mg} \mathrm{m}^{-2}\right.$ per day), mitomycin C ( $4.5 \mathrm{mg} \mathrm{m}^{-2}$ per day), bleomycin $\left(15 \mathrm{mg} \mathrm{m}^{-2}\right.$ per day), cisplatin (35 $\mathrm{mg} \mathrm{m}^{-2}$ per day), and 5-fluorouracil (1200 $\mathrm{mg} \mathrm{m}^{-2}$ per day) (Roth et al, 2000). These drugs were infused continuously by using pump for 2 days in each course. Besides, leucovorin (50 mg per day) was administered intravenously for 2 days. If the serum creatinine was more than $2.0 \mathrm{mg} \mathrm{dl}^{-1}$, a $25 \%$ dosage reduction was required and cisplatin was replaced by carboplatin ( $100 \mathrm{mg} \mathrm{m}^{-2}$ per day). The course was repeated with an interval of 4 weeks.

Assessment of chemotherapy response. Tumour responses were assessed by visual inspection at each visit and by using Response Evaluation Criteria in Solid Tumours (RECIST) criteria (Therasse et al, 2000). Disappearance of primary lesion without lymphadenopathy $>1.0 \mathrm{~cm}$ was considered complete response (CR). Partial response (PR) was defined as at least a $30 \%$ decrease in the sum of the longest diameter of target lesions with or without residual lymphadenopathy $>1.0 \mathrm{~cm}$. Progressive disease (PD) was defined as at least a $20 \%$ increase in the sum of the longest diameter of target lesions. Tumour response that did not meet these criteria was defined as stable disease (SD). The IA chemotherapy-related toxicity was graded by the National Cancer Institute (NCI) Common Terminology Criteria for Adverse Events (CTCAE) v3.0.

Surgical decision. After neoadjuvant IA chemotherapy, patients with residual tumours underwent partial or total penectomy depending on tumour stage. A tumour-free margin of $5 \sim 10 \mathrm{~mm}$ was needed for partial penectomy. If the patient achieved complete response, incisional biopsy of previous lesion was performed to confirm no residual tumour. Regarding the inguinal lymph node, routine inguinal lymphadenectomy (LAD) was performed in case with palpable inguinal lymph node. For nonpalpable inguinal node, inguinal LAD was only performed in patients with T1G2 tumour or higher stage.

Statistical analysis. Recurrence-free survival was defined as the date of curative surgery to the last date of follow-up or recurrence. For complete responder without further surgery, the recurrencefree survival was defined as the date of negative biopsy to the last date of follow-up or recurrence. Survival was estimated by using the Kaplan-Meier method. For further analysis, patients were divided into two groups: node-negative penile SCC and nodepositive penile SCC. Study groups were compared by means of the log-rank test. Statistical analyses were calculated by using the SPSS Base 17.0 (SPSS Inc., Chicago, IL, USA). A $P$-value of $<0.05$ was considered statistically significant.

\section{RESULTS}

Patient characteristics. A total of 12 patients with histopathologically proven SCC of the penis undergoing IA chemotherapy were included in this study (Table 1). Of the 12 patients, 5 had palpable inguinal lymph node at initial diagnosis. Of these 12 patients, only one patient (case 8 ) had prior surgery at initial interview, whereas the others had no treatment before IA chemotherapy. In case 8 , he had undergone total penectomy with perineal urethrostomy because of T3 tumour at other hospital in February 2011. However, poor healing of perineal wound was noted after surgery and then he was referred to our hospital for further management. Biopsy of perineal wound was done in May 2011 and pathological examination revealed residual cancer. Thereafter, he received two cycles of IA chemotherapy and then underwent total urethrectomy with removal of prostate (Figure 1).

IA chemotherapy and tumour response. One patient (case 1) had impaired renal function and he received carboplatin in combination with other four agents. The remaining 11 patients all received cisplatin-based combination chemotherapy. Two patients only received one cycle of neoadjuvant IA chemotherapy because of

\begin{tabular}{|c|c|c|c|c|c|c|c|c|c|}
\hline $\begin{array}{l}\text { Case } \\
\text { no. }\end{array}$ & Age & $\begin{array}{l}\text { TNM at } \\
\text { diagnosis }\end{array}$ & $\begin{array}{l}\text { SCC } \\
\text { differentiation }\end{array}$ & $\begin{array}{l}\mathrm{IA} \mathrm{C/T} \\
\text { cycle }\end{array}$ & Response & Curative surgery & $\begin{array}{l}\text { F/U duration } \\
\text { (month) }\end{array}$ & $\begin{array}{l}\text { Treatment after } \\
\text { recurrence }\end{array}$ & Status \\
\hline 1 & 56 & T2NOMO & Good & 4 & PR & $\mathrm{Nil}$ & Loss of F/U & & Unknown \\
\hline 2 & 70 & T1NOM0 & Good & 5 & $\mathrm{CR}$ & $\begin{array}{l}\text { PP because of } \\
\text { recurrence at } 8 \text { months }\end{array}$ & 88 & NR after PP & Alive \\
\hline 3 & 61 & T1N0M0 & Good & 3 & PR & PP & 72 & NR & Alive \\
\hline 4 & 48 & $\mathrm{~T} 2 \mathrm{~N} 2 \mathrm{M} 0$ & Moderate & 3 & PR & $P P+$ inguinal LAD & 34 & IA C/T and RT & DOD \\
\hline 5 & 79 & $\mathrm{~T} 2 \mathrm{~N} 2 \mathrm{MO}$ & Moderate & 3 & PR & $P P+$ inguinal LAD & 21 & $\mathrm{IA} \mathrm{C} / \mathrm{T}$ and $\mathrm{RT}$ & DOD \\
\hline 6 & 76 & T3N2M0 & Moderate & 2 & SD & $T P+$ inguinal LAD & 12 & $\mathrm{IA} \mathrm{C} / \mathrm{T}$ and $\mathrm{RT}$ & Alive \\
\hline 7 & 64 & T3N1M0 & Moderate & 2 & PR & TP + inguinal LAD & 49 & $\mathrm{IA} \mathrm{C/T}$ and $\mathrm{RT}$ & Alive \\
\hline 8 & 33 & T3NOMO & Good & 2 & CR & TUx + inguinal LAD & 32 & NR & Alive \\
\hline 9 & 67 & T1N0M0 & Good & 2 & $\mathrm{CR}$ & $\mathrm{Nil}$ & 14 & NR & Alive \\
\hline 10 & 70 & $\mathrm{~T} 2 \mathrm{~N} 1 \mathrm{M} 0$ & Moderate & 1 & SD & TP + inguinal LAD & 10 & NR & Alive \\
\hline 11 & 89 & T3NOMO & Moderate & 1 & PR & TP + inguinal LAD & 8 & NR & Alive \\
\hline 12 & 51 & T1N0M0 & Good & 2 & CR & $\mathrm{Nil}$ & 12 & NR & Alive \\
\hline
\end{tabular}




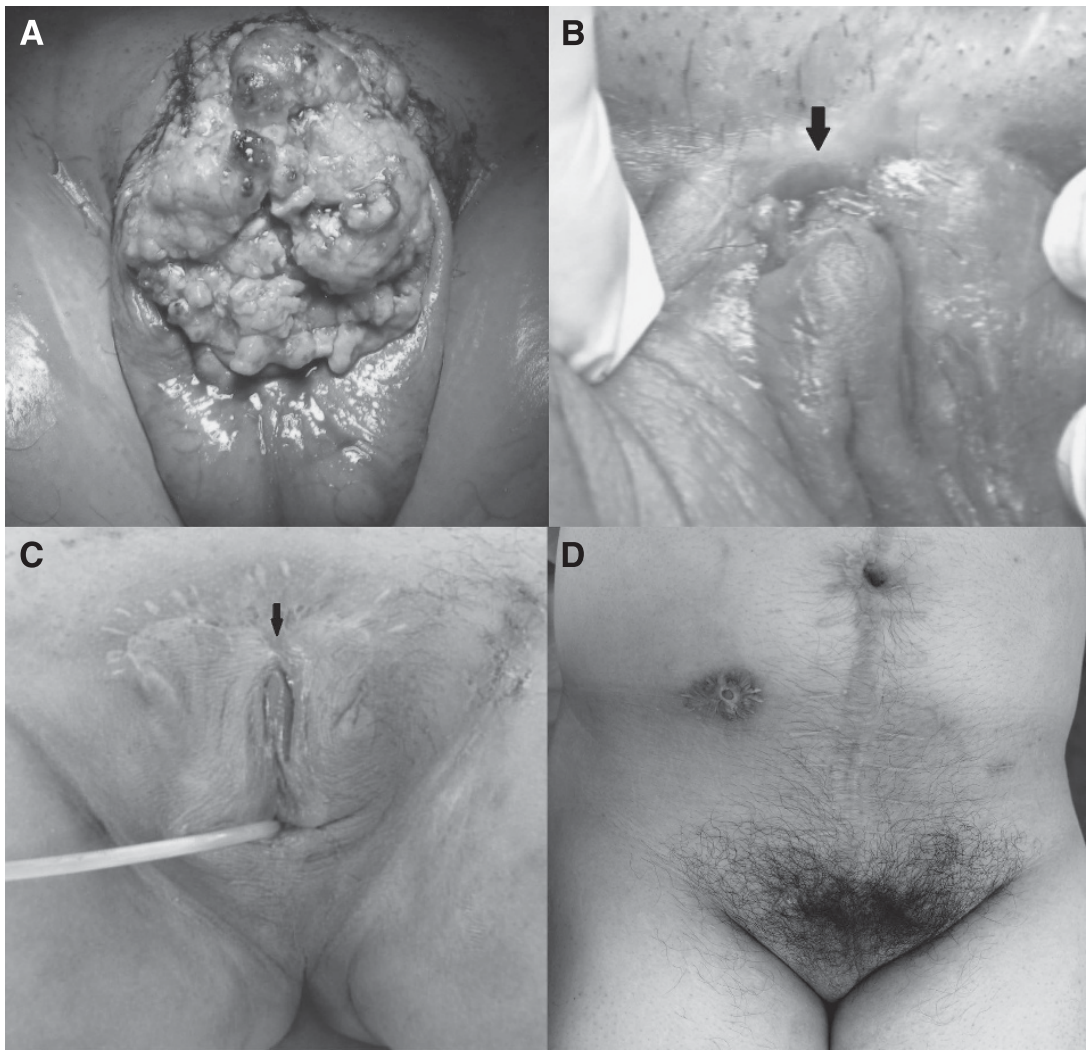

Figure 1. (A) Case 8 was presented with T3 penile SCC at diagnosis. (B) Total penectomy was performed at other hospital. Residual tumour (arrow) was noted at surgical wound. (C) After IA chemotherapy, no residual tumour was found. (arrow). (D) Hyperpigmentation over lower abdomen and external genitalia has been still present at 3-year follow-up.

tumour pain and they asked for surgery as soon as possible. No one discontinued IA chemotherapy because of intolerance of toxicity. An objective tumour response was noted in 10 of 12 patients (83\%), consisting of $4 \mathrm{CR}$ and 6 PR. Stable disease was found in two patients. In node-negative patients $(n=7)$, the response rate was $100 \%$ (4 CR and $3 \mathrm{PR}$ ).

Toxicity of IA chemotherapy. Anorexia was the most common adverse event of IA chemotherapy in most cases ( 7 out of $12,58 \%$ ) and usually was tolerable (grade 1 or 2) (Table 2). Three patients experienced grade 3 haematological toxicity. They were treated with blood transfusion or intravenous antibiotic therapy.

Surgery. An overview of treatment modalities and outcomes is shown in Table 1 and Figure 2. Of the seven patients without palpable inguinal node, two of three partial responders underwent partial penectomy after neoadjuvant IA chemotherapy and there is no recurrence to date after surgery (Figure 2). Of the complete responders, one (case 8) underwent total urethrectomy with removal of prostate, and pathology showed no residual tumour. He is still alive and disease-free for nearly 3 years till now. Another complete responder (case 2) had tumour recurrence 8 months after last cycle of IA chemotherapy and then he underwent partial penectomy. The remaining two complete responders had no further treatment, and are in regular follow-up without recurrence.

Of the five patients with palpable inguinal node (three PR and two SD), all of them received surgery with curative intent (total or partial penectomy with inguinal LAD). However, there were still four patients who had inguinal nodal recurrence and required subsequent salvage IA chemotherapy and radiotherapy. Two of them are still ongoing treatment but the other two had died of disease.
Recurrence-free survival. One patient (case 1) was excluded for analysis because of loss of follow-up. The remaining 11 patients were divided into node-negative $(n=6)$ and node-positive $(n=5)$ groups. The recurrence-free survival was in favour of nodenegative group (log-rank test, $P=0.041$; Figure 3 ).

\section{DISCUSSION}

The role of IA chemotherapy in the treatment of penile cancer is not well defined. Because of the rarity of this cancer, only several small series have reported the outcome of IA chemotherapy with single agent or combination regimens for penile cancer treatment. To our knowledge, this is the largest report on IA chemotherapy in penile SCC. Our result showed that IA chemotherapy is feasible in penile SCC with excellent response rate, especially in patients without nodal invasion. With the cosmetic or functional concern, sometimes patients with penile cancer may hesitate for penectomy. This treatment provides the possibility of preserving penis in patients achieving complete response. According to EAU guideline, chemotherapy was only used in T4 penile cancer (Pizzocaro et al, 2010). In this study, IA chemotherapy was used not only in advanced penile cancer but also in T1-T2 tumours before surgery. This strategy effectively shrunk the tumour burden and reduced the resected penile length. Using IA chemotherapy as first-line therapy before surgery, this provides a new insight of organ preservation.

The rationale of IA chemotherapy in penile cancer is to deliver higher concentration of anticancer drugs to the pelvic region with less systemic exposure. Theoretically, IA chemotherapy has the benefit over intravenous access of chemotherapy in view of drug concentration and toxicity (Robbins et al, 1992). Someone may 
question whether the concentration of regimens in target organ is really higher via intraarterial access than that via intravenous access. In the current study, we did not directly compare the drug concentration in target region between the two accesses. However, a previous study reported that well-stained areas represented the areas with good perfusion of drugs (Galmarini et al, 2000). In contrast to well-stained areas, areas with less stain were considered less exposed to chemotherapeutic drugs. In case 8 , hyperpigmentation over lower abdomen and external genitalia was noted after IA chemotherapy (Figure 1D) and this may provide an alternative evidence of higher concentration of chemotherapy regimens to pelvic area.

Roth et al (2000) first reported the use of methotrexate, mitomycin C, bleomycin, cisplatin, and 5-fluorouracil in the treatment of locally advanced or recurrent penile cancer by intraarterial route. Of the seven patients evaluable for response, the objective response rate was $86 \%$ (three CR and three PR). Liu et al (2013) also used intraarterial chemotherapy with gemcitabine and

Table 2. The adverse events of intraarterial chemotherapy in patients with penile squamous cell carcinoma (SCC)

\begin{tabular}{|l|c|c|c|c|}
\hline \multicolumn{5}{|c|}{ No. of patients by grade } \\
\hline Toxicity & Grade 1 & Grade 2 & Grade 3 & Grade 4 \\
\hline Anorexia & 2 & 5 & 0 & 0 \\
\hline Anaemia & 0 & 0 & 2 & 0 \\
\hline Febrile neutropenia & 0 & 0 & 1 & 0 \\
\hline Fatigue & 1 & 0 & 0 & 0 \\
\hline
\end{tabular}

cisplatin to treat locally advanced or recurrent penile SCC. In their series, an overall response rate of $83 \%$ was achieved. The most common toxicity was leukocytopenia (25\%), followed by nausea/ vomiting, anorexia, and anaemia. None died of chemotherapyrelated toxicity. The response rates in these studies are similar to ours.

Sheen et al (2010) reported a series of 6 patients treated with methotrexate alone for penile verrucous carcinoma. Their result showed a $100 \%$ response rate (4 CR and 2 PR) and tolerable toxicity in this type of malignancy. Verrucous carcinoma represents an extremely well-differentiated variant of SCC. It is characterised by local invasive behaviour but rarely metastasises. Different from their series, all the patients included in our study were pathologically proven classic SCC that represents more aggressive and metastatic tumour behaviour. Considering that, we used the combination regimens for penile SCC treatment. However, it remains controversial whether the single regimen could bring the similar efficacy in penile SCC because of the lack of prospective study comparing single regimen with multiple regimens.

With regard to the safety of IA chemotherapy, the most frequent adverse event was anorexia. Only three patients experienced grade 3 haematological toxicity and none died of toxicity. The Southwest Oncology Group used the combination regimens (cisplatin/ bleomycin/methotrexate) as systemic chemotherapy to treat penile cancer with only an overall response rate of $32.5 \%$ (Haas et al, 1999). Of the 41 evaluable patients, 5 patients died of chemotherapy-related toxicity and 6 patients suffered from life-threatening adverse events (Haas et al, 1999). Leijte et al (2007) also reported a series of 20 patients receiving neoadjuvant chemotherapy for unresectable penile cancer. In their study, three patients suffered from chemotherapy-related toxic death. On the whole, IA chemotherapy-related toxicity was mild and tolerable.

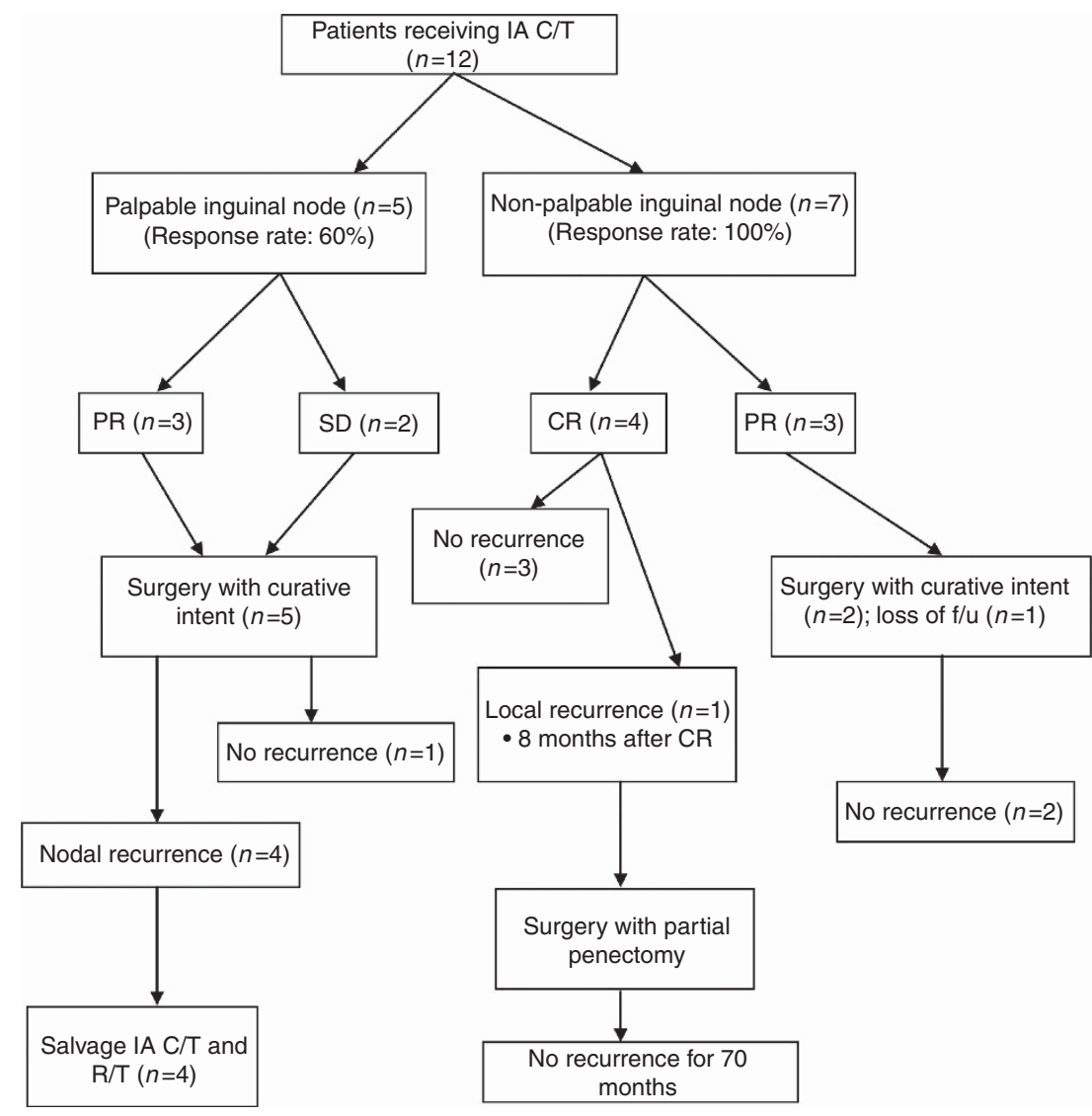

Figure 2. Overview of treatment modalities and outcome. Abbreviations: $C R=$ complete response; $\mid \mathrm{A} C / \mathrm{T}=$ intraarterial chemotherapy; $\mathrm{PR}=$ partial response; $\mathrm{R} / \mathrm{T}=$ radiotherapy; $\mathrm{SD}=$ stable disease. 


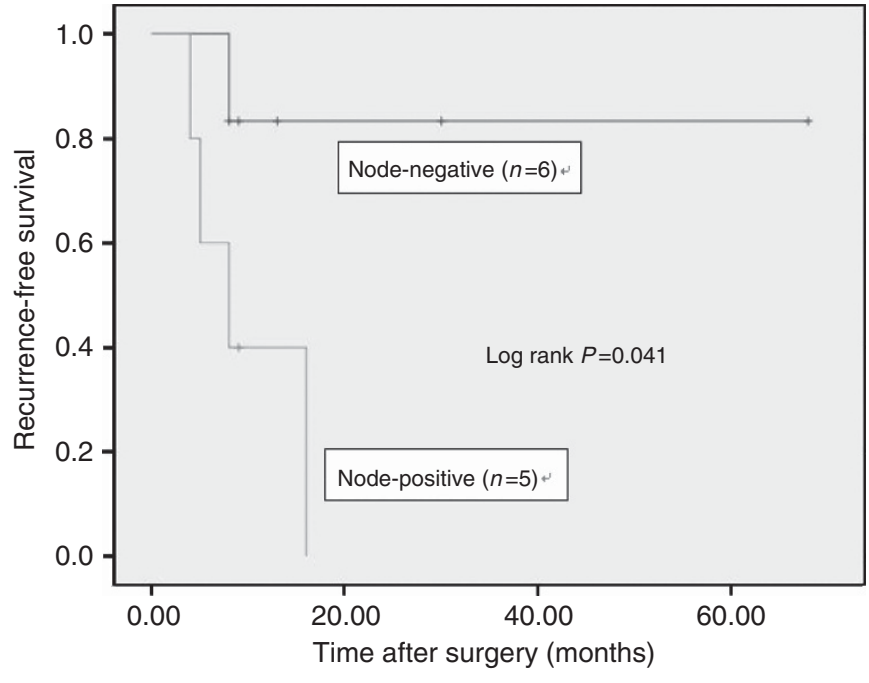

Figure 3. Recurrence-free survival after IA chemotherapy and curative surgery. Comparison between the patients with initial nodal invasion and those without nodal invasion.

Because of the rarity of penile cancer, it is difficult to prospectively compare the efficacy and toxicity between different therapeutic agents. Thus, the best chemotherapeutic regimen for penile SCC is still under investigation. It may be helpful to learn the experience in other fields of SCC as a rationale, such as head and neck, because the natural history and clinical behaviour of penile SCC is similar to that of SCC of the head and neck (Pizzocaro et al, 1997). In a phase II prospective study, Pagliaro et al (2010) used paclitaxel, ifosfamide, and cisplatin (TIP), previously proposed by Shin et al (1998) for recurrent or metastatic head and neck SCC, as neoadjuvant chemotherapy to treat stage $\mathrm{N} 2$ or $\mathrm{N} 3$ penile cancer. Their result showed a response rate of $50 \%$ and overall survival was significantly associated with the chemotherapy response (Pagliaro et al, 2010).

Recent studies have shown that induction chemotherapy with taxanes in combination with cisplatin and fluorouracil provided higher complete response rate, longer survival, and reduced toxic events in the SCC of head and neck when compared with standard regimens with cisplatin and fluorouracil (Posner et al, 2007; Vermorken et al, 2007). Pizzocaro et al (2009) reported a series of six patients receiving neoadjuvant systemic chemotherapy with three-drug combination (taxanes, cisplatin, and fluorouracil) before surgery for unresectable or node-recurrence penile SCC. The response rate (four CR and one PR) was surprising and only one had severe nausea and vomiting during chemotherapy. With the help of the experience, new trial with taxanes-combined IA chemotherapy for penile SCC may be further studied to evaluate the efficacy and safety.

There are some limitations in this study. First, this is a smallscale and retrospective study because penile SCC is an extremely rare cancer in our country. We did not compare the outcome of neoadjuvant IA chemotherapy followed by surgery with that of surgery alone in node-negative patients. However, at least IA chemotherapy provides the possibility of cosmetic and functional preservation if achieving complete response. Second, the standard protocol that how many cycles of IA chemotherapy will be needed is still uncertain at present. In the previous article, we observed that the tumour response was usually quick and dramatic after two to three cycles of IA chemotherapy (Chen et al, 2009). Therefore, at least two cycles of IA chemotherapy could be considered before surgery in case of no severe adverse events.

In conclusion, the preliminary results showed that IA chemotherapy could be used as first-line therapy in any stage of penile SCC before surgery. This strategy effectively shrunk the tumour burden and reduced the resected penile length. This procedure is simple and feasible with low toxicity and excellent response rate for penile SCC. However, the optimal agents and treatment cycles should be further determined in large-scale study.

\section{CONFLICT OF INTEREST}

The authors declare no conflict of interest.

\section{REFERENCES}

Chen $\mathrm{CH}$, Kang $\mathrm{CH}$, Chiang $\mathrm{PH}$ (2009) Intra-arterial infusion of chemotherapy in the treatment of penile cancer. Jpn J Clin Oncol 39: 825-828.

Culkin DJ, Beer TM (2003) Advanced penile carcinoma. J Urol 170: 359-365. Galmarini FC, Galmarini CM, Sarchi MI, Abulafia J, Galmarini D (2000) Heterogeneous distribution of tumor blood supply affects the response to chemotherapy in patients with head and neck cancer. Microcirculation 7: 405-410.

Haas GP, Blumenstein BA, Gagliano RG, Russell CA, Rivkin SE, Culkin DJ, Wolf M, Crawford ED (1999) Cisplatin, methotrexate and bleomycin for the treatment of carcinoma of the penis: a Southwest Oncology Group study. J Urol 161: 1823-1825.

Leijte JA, Kerst JM, Bais E, Antonini N, Horenblas S (2007) Neoadjuvant chemotherapy in advanced penile carcinoma. Eur Urol 52: 488-494.

Liu JY, Li YH, Liu ZW, Zhang ZL, Ye YL, Yao K, Han H, Qin ZK, Zhou FJ (2013) Intraarterial chemotherapy with gemcitabine and cisplatin in locally advanced or recurrent penile squamous cell carcinoma. Chin J Cancer 32: 619-623.

Pagliaro LC, Williams DL, Daliani D, Williams MB, Osai W, Kincaid M, Wen S, Thall PF, Pettaway CA (2010) Neoadjuvant paclitaxel, ifosfamide, and cisplatin chemotherapy for metastatic penile cancer: a phase II study. J Clin Oncol 28: 3851-3857.

Pizzocaro G, Algaba F, Horenblas S, Solsona E, Tana S, Van Der Poel H, Watkin NA (2010) European Association of Urology (EAU) Guidelines Group on Penile Cancer. EAU penile cancer guidelines 2009. Eur Urol 57: 1002-1012.

Pizzocaro G, Nicolai N, Milani A (2009) Taxanes in combination with cisplatin and fluorouracil for advanced penile cancer: preliminary results. Eur Urol 55: 546-551.

Pizzocaro G, Nicolai N, Piva L (1997) Chemotherapy for cancer of the penis. In Principles and Practice of Genitourinary Oncology, Raghavan D, Leibel SA, Scher HI, Langhe P (eds). Lippincot-Raven Publishers: Philadelphia, PA, pp 973-977.

Posner MR, Hershock DM, Blajman CR, Mickiewicz E, Winquist E, Gorbounova V, Tjulandin S, Shin DM, Cullen K, Ervin TJ, Murphy BA, Raez LE, Cohen RB, Spaulding M, Tishler RB, Roth B, Viroglio Rdel C, Venkatesan V, Romanov I, Agarwala S, Harter KW, Dugan M, Cmelak A, Markoe AM, Read PW, Steinbrenner L, Colevas AD, Norris Jr CM, Haddad RI. TAX 324 Study Group (2007) Cisplatin and fluorouracil alone or with docetaxel in head and neck cancer. N Engl J Med 357: 1705-1715.

Robbins KT, Storniolo AM, Kerber C, Seagren S, Berson A, Howell SB (1992) Rapid superselective high-dose cisplatin infusion for advanced head and neck malignancies. Head Neck 14: 364-371.

Roth AD, Berney CR, Rohner S, Allal AS, Morel P, Marti MC, Aapro MS, Alberto P (2000) Intra-arterial chemotherapy in locally advanced or recurrent carcinomas of the penis and anal canal: an active treatment modality with curative potential. Br J Cancer 83: 1637-1642.

Sheen MC, Sheu HM, Jang MY, Chai CY, Wang YW, Wu CF (2010) Advanced penile verrucous carcinoma treated with intra-aortic infusion chemotherapy. J Urol 183: 1830-1835.

Shin DM, Glisson BS, Khuri FR, Ginsberg L, Papadimitrakopoulou V, Lee JJ, Lawhorn K, Gillenwater AM, Ang KK, Clayman GL, Callender DL, Hong WK, Lippman SM (1998) Phase II trial of paclitaxel, ifosfamide, and cisplatin in patients with recurrent head and neck squamous cell carcinoma. J Clin Oncol 16: 1325-1330.

Tanaka K, Yabushita Y, Nakagawa K, Kumamoto T, Matsuo K, Taguri M, Endo I (2013) Debulking surgery followed by intraarterial 5-fluorouracil 
chemotherapy plus subcutaneous interferon alfa for massive hepatocellular carcinoma with multiple intrahepatic metastases: A pilot study. Eur J Surg Oncol 39: 1364-1370.

Therasse P, Arbuck SG, Eisenhauer EA, Wanders J, Kaplan RS, Rubinstein L, Verweij J, Van Glabbeke M, van Oosterom AT, Christian MC, Gwyther SG (2000) New guidelines to evaluate the response to treatment in solid tumors. European Organization for Research and Treatment of Cancer, National Cancer Institute of the United States, National Cancer Institute of Canada. J Natl Cancer Inst 92: 205-216.

Vermorken JB, Remenar E, van Herpen C, Gorlia T, Mesia R, Degardin M, Stewart JS, Jelic S, Betka J, Preiss JH, van den Weyngaert D, Awada A, Cupissol D, Kienzer HR, Rey A, Desaunois I, Bernier J, Lefebvre JL (2007) Cisplatin, fluorouracil and docetaxel in unresectable head and neck cancer. N Engl J Med 357: 1695-1704.
Wu CF, Chen CM, Chen CH, Shieh TY, Sheen MC (2007) Continuous intraarterial infusion chemotherapy for early lip cancer. Oral Oncol 43: 825-830.

Wu CF, Chen CM, Shen YS, Huang IY, Chen CH, Chen CY, Shieh TY, Sheen MC (2008) Effective eradication of oral verrucous carcinoma with continuous intraarterial infusion chemotherapy. Head Neck. 30: 611-617.

Zhang W, Liu R, Wang Y, Qian S, Wang J, Yan Z, Zhang H (2013) Efficacy of intraarterial chemoinfusion therapy for locally advanced breast cancer patients: a retrospective analysis of 28 cases. Onco Targets Ther 6: 761-765.

This work is published under the standard license to publish agreement. After 12 months the work will become freely available and the license terms will switch to a Creative Commons AttributionNonCommercial-Share Alike 3.0 Unported License. 